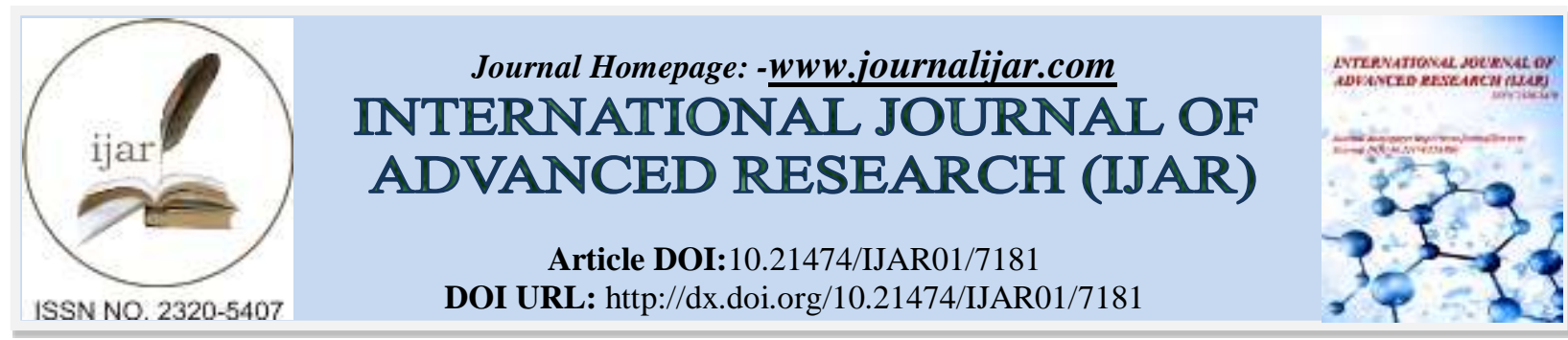

RESEARCH ARTICLE

\title{
DEVELOPMENT AND ASSESSMENT OF A COAGGLUTINATION TEST FOR DETECTING CANINE PARVOVIRUS IN CLINICAL SPECIMENS.
}

\author{
Ahlam Kadiri ${ }^{1}$, Nadia Amrani ${ }^{1}$, Khalil Zro ${ }^{2}$ and Jaouad Berrada ${ }^{1}$. \\ 1. Institut Agronomique et Vétérinaire Hassan II. \\ 2. Société de Productions Biologiques et Parmaceutiques Vétérinaires (Biopharma).
}

\section{Manuscript Info}

Manuscript History

Received: 02 April 2018

Final Accepted: 04 May 2018

Published: June 2018

Keywords:-

dogs, canine parvovirus, rapid diagnostic, Coagglutination test.

\begin{abstract}
Rapid diagnosis of canine parvovirus (CPV-2) infection is of key importance in management and control of the disease. The present study aims at the development and evaluation of an easy to use rapid test for the detection of canine parvovirus particles in clinical samples collected and tested during daily veterinary practice.

For this purpose, the coagglutination test (COA) using Methylene blue stained anti-CPV2 antibodies sensitised staphylococcus aureus ATCC 12598 was performed to detect CPV-2 in 91 clinical samples (86 rectal swabs and 5 organ homogenates) collected between 2011 and 2015 from suspected canine clinical cases received in Moroccan veterinary clinics. The same samples were previously tested by Hemagglutination test and real-time PCR (Amrani et al., 2016), the results were compared and employed in the evaluation of COA.

The results of the COA showed $79.12 \%$ (72/91) of tested samples to be positive and $20.87 \%$ (19/91) to be negative. The intensity of reaction in positive samples was scored high (3+) in 6 samples (6.6\%), medium $(2+)$ in 28 samples $(30.7 \%)$ and low (1+) in 57 samples (62.6\%) which occurred within a median time of $3 \mathrm{~min}, 4 \mathrm{~min}$ and $3 \mathrm{~min} 42 \mathrm{~s}$, respectively. In comparison with PCR, the COA test demonstrated a good agreement of $80.2 \%$ and a sensitivity of $80 \%$. The coagglutination test is a promising test allowing daily diagnostic of canine parvovirus in field, veterinary clinics and non- equipped laboratories with molecular techniques.
\end{abstract}

Copy Right, IJAR, 2018,. All rights reserved.

\section{Introduction:-}

Digestive disorders including diarrhoea and vomiting are considered as major canine health problems and the most frequent cause for veterinary consultation (Rakha et al., 2015; Ylmaz et al., 2002). Among numerous aetiologies involved in these disorders, canine parvovirus (CPV-2) infection is the most frequent and serious pathology, characterized by high morbidity and mortality rates, especially in susceptible puppies of 6 weeks to 6 months of age (Prittie, 2004). The causative CPV-2 is a non-enveloped virus with icosahedral capsid enclosing a single stranded DNA of $~ 5000$ bp characterized by a high mutation rate similar to that observed for RNA viruses (Decaro et al., 2009). Since its emergence, CPV-2 has rapidly evolved giving rise to highly pathogenic variants CPV-2a, $2 \mathrm{~b}$ and $2 \mathrm{c}$ (Decaro and Buonavoglia, 2012). Vaccination remains the main tool to control and prevent canine parvovirus 
infection. However, vaccine neutralisation by long-lasting maternal antibodies (MDA) associated with poor or absence of cross-protection between CPV-2 antigenic variants, compromise seriously the success of vaccination (Decaro et al., 2005a, 2008). Early, rapid, accurate diagnosis and management of CPV-2 is of key importance in effective reduction of infected dog's morbidity and mortality (Kim et al., 2015). Molecular diagnostic tests, notably the real-time PCR, were demonstrated to be more sensitive than traditional methods (Decaro et al., 2005b; Desario et al., 2005). However, PCR-based tests are expensive, carried out in specialized laboratories only and are difficult to establish for standard practice in veterinary clinics. Therefore, development of rapid, simple, sensitive and affordable tests detecting CPV-2 particles in clinical samples would be convenient and can be conducted in veterinary clinical practice (Kantere et al., 2015). The coagglutination (COA) test was previously developed (Genovn and Ivanov, 2006; Singh et al., 1998) by exploiting the property of staphylococcus aureus membrane protein A to bind the Fc portion of anti-parvovirus IgG which recognizes CPV-2 antigen in positive tested samples. Compared with Haemagglutination test (HA), this test was reported specific and sensitive (Genovn and Ivanov, 2006).

The aim of the present study is to develop an easy to use and interpret coagglutination assay and to evaluate its performances (sensitivity, positive predictive value, overall agreement) in comparison with real-time PCR as gold standard test for canine parvovirus detection.

\section{Material and Methods:- \\ Clinical samples:-}

A total of 91 clinical samples consisting of 86 rectal swabs and 5 organ's homogenates were collected from suspected dogs suffering from diarrhoea and vomiting, received in veterinary clinics located in Marrakech, Settat, Khemisset, Casablanca, Temara, Rabat, Sale and Machraa Belakssiri, between 2011 and 2015. These samples were previously processed and screened for canine parvovirus infection by means of real-time PCR and HA (Amrani et al., 2016) (table1).

\section{Bacteria:-}

Lyophilised Staphylococcus aureus ATCC 12598 was reconstituted and revived following the manufacturer's recommendations. An isolated $S$. aureus colony was cultured into Brain and Heart broth (BHB) for $18 \mathrm{~h}$ at $37^{\circ} \mathrm{C}$ in a shaking water bath. The resulting bacterial culture was washed 3 times in PBS and inactivated by adding $0.5 \%$ formaldehyde solution at $4{ }^{\circ} \mathrm{C}$ for $18 \mathrm{~h}$ and washed and heated at $80^{\circ} \mathrm{C}$ for $20 \mathrm{~min}$. Following inactivation, bacteria were washed three times and resuspended in PBS containing $0.5 \%$ Tween 20 . Using spectrophotometry at $250 \mathrm{~nm}$, bacterial absorbance was fixed at 0.69 , corresponding to $2.3 \times 10^{10} \mathrm{cells} / \mathrm{ml}$. This suspension was stored at $4{ }^{\circ} \mathrm{C}$ for maximum 3 months (Montassier et al., 1994).

\section{Production of hyper immune serum:-}

As shown in figure 1, Anti-parvovirus hyperimmune serum was produced on SPF, New Zealand, male rabbit weighting $3.5 \mathrm{~kg}$. Briefly, three CPV-2 vaccine doses (Primodog ${ }^{\circledR}$, CPV strain C-780916 $\geq 105^{.5}$ DICC $_{50}$ ) were inoculated by subcutaneous route on day 0,15 and 30, respectively. At day 40, blood was collected from the hyperimmunized rabbit and serum was separated by centrifugation. After decomplementation at $56^{\circ} \mathrm{C}$ for 30 min, hyperimmune serum was mixed with the previously described $S$ aureus suspension at 2:1 (V/V) and incubated for $3 \mathrm{~h}$ at room temperature. To facilitate the test lecture, IgG-S.Aureus complexes were stained using $0.075 \%$ methylen blue stain. The produced reagent was stored at $4^{\circ} \mathrm{C}$ for use during one month.

\section{Test procedure:-}

The coagglutination test was performed using a glass slide by homogenising equal volumes $(50 \mu \mathrm{l})$ of clinical samples supernatants with stained $\mathrm{IgG}-\mathrm{S}$. Aureus reagent according to the method described previously (Genovn and Ivanov, 2006). The slide was examined macroscopically during $10 \mathrm{~min}$ for development of agglutinin particles. The time and the intensity of the reaction $(1+, 2+, 3+)$ were recorded (figure 2). Positive and negative controls consisting of a positive and negative real-time PCR fecal samples collected from dogs were used. The overall procedure is summarised in figure 1. 


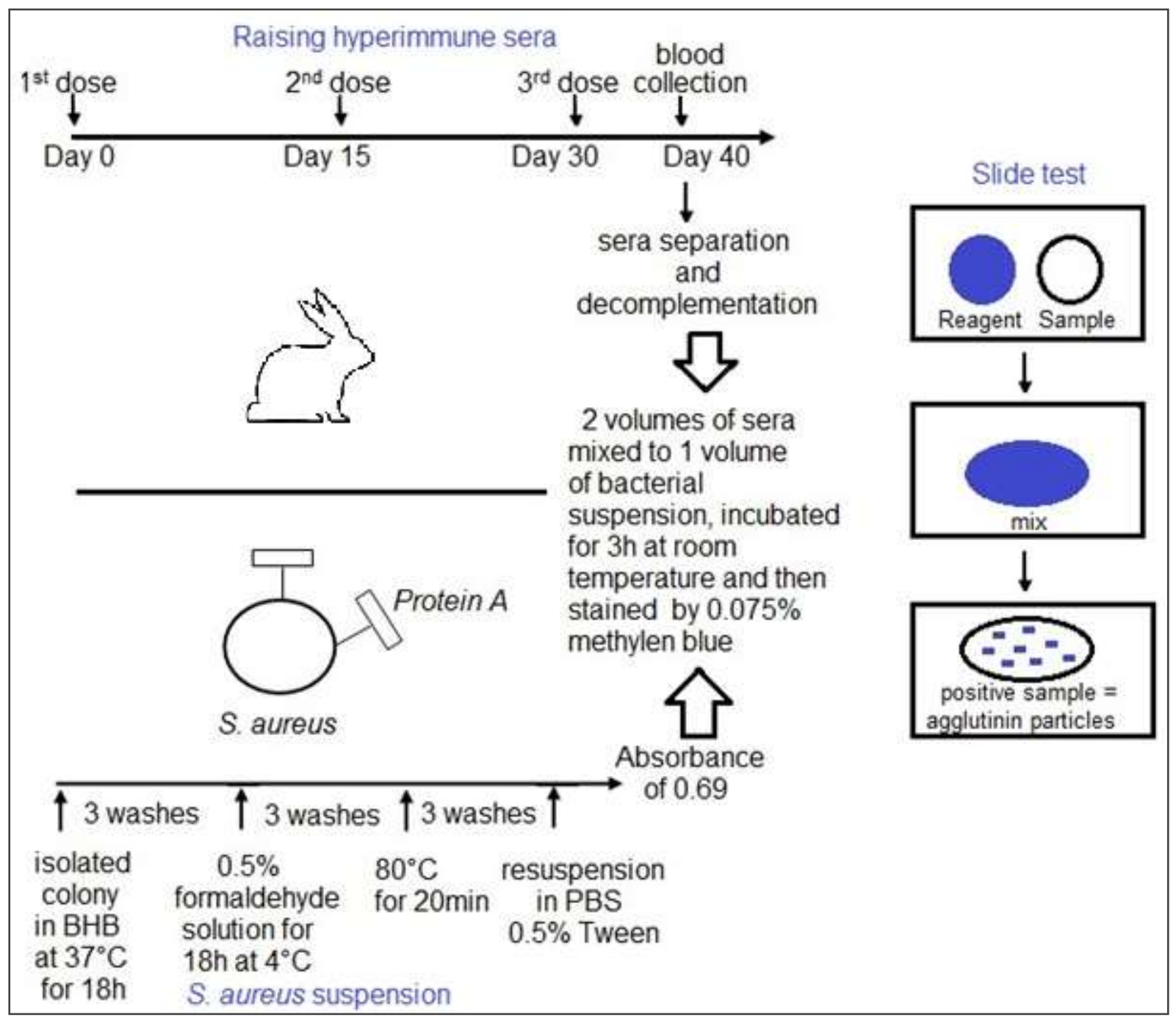

Figure 1:-Reagent preparation and specimen testing protocol

The test performances were evaluated by calculating sensitivity and overall agreement in comparison with the gold standard test, real-time PCR, carried out previously for the same samples (Amrani et al., 2016).

\section{Results:-}

Obtained results and information about tested samples are presented in table 1.

Among 91 tested samples, the coagglutination test detected 72 positive samples $(79.12 \%)$ and 19 negative samples (20.87\%). Agglutinin particles appeared within 45 s to $10 \mathrm{~min}$ after mixing the reagent with samples (figure2). The intensity of reaction and time of its appearance was scored from $1+$ to $3+$ and in seconds, respectively. Agglutinin particles concentration was high (3+) in 6 samples (6.6\%), median (2+) in 28 samples (30.7\%) and low (1+) in 57 samples $(62.6 \%)$.

Positive reactions occurred within a median time of $3 \mathrm{~min}, 4 \mathrm{~min}$ and $3 \min 42 \mathrm{~s}$ respectively (figure 3 ). Sensitivity of the test reached $80 \%$ (72/90) and overall agreement accounted for $80.2 \%$ between COA and PCR tests (73/91). 
Table 1:-Informations regarding samples, DNA loads using real-time PCR, scored COA intesity and reaction time

\begin{tabular}{|c|c|c|c|c|c|c|c|c|c|c|}
\hline Code & Sample & Year & $\begin{array}{l}\text { Age by } \\
\text { month }\end{array}$ & Breed & Sex & Vaccination & Origin & $\begin{array}{l}\text { Real-time } \\
\text { PCR } \\
\text { (DNA } \\
\text { copy/gm) }\end{array}$ & $\begin{array}{l}\text { COA } \\
\text { intensi } \\
\text { ty }\end{array}$ & $\begin{array}{l}\text { Reaction } \\
\text { time }\end{array}$ \\
\hline Alg-11 & $\mathrm{I}$ & 2011 & NA & NA & NA & NA & Marrakech & $1.1 \times 10^{5}$ & $1+$ & $3 \mathrm{~min} 40 \mathrm{~s}$ \\
\hline S10-11 & I & 2011 & 2 & $\mathrm{R}$ & $\mathrm{F}$ & UV & Rabat & $1.7 \times 10^{8}$ & $1+$ & $3 \min 25 s$ \\
\hline Dark11 & $\mathrm{S}$ & 2011 & NA & NA & NA & NA & NA & $2.7 \times 10^{7}$ & $\mathrm{~N}$ & - \\
\hline Parvo 3 & $\mathrm{I}$ & 2012 & 1 & $\mathrm{R}$ & $\mathrm{F}$ & UV & Rabat & $1.3 \times 10^{8}$ & $1+$ & $3 \min$ \\
\hline Parvo 4 & I & 2012 & NA & NA & NA & NA & Rabat & $3.8 \times 10^{6}$ & $1+$ & $3 \mathrm{~min}$ \\
\hline P01-13 & $\mathrm{F}$ & 2013 & 3 & $\mathrm{P}$ & $\mathrm{F}$ & $\mathrm{V}$ & Rabat & $1,3 \times 10^{9}$ & $2+$ & $4 \mathrm{~min} 05 \mathrm{~s}$ \\
\hline $\mathrm{P} 02-13$ & $\mathrm{~F}$ & 2013 & 4 & LR & $\mathrm{M}$ & $\mathrm{V}$ & Rabat & $2.8 \times 10^{9}$ & $\mathrm{~N}$ & - \\
\hline P03-13 & $\mathrm{I}$ & 2013 & 6 & LR & $\mathrm{F}$ & $\mathrm{V}$ & Rabat & $4.6 \times 10^{5}$ & $1+$ & $3 \min 55 \mathrm{~s}$ \\
\hline P04-13 & $\mathrm{F}$ & 2013 & 6 & GR & $\mathrm{F}$ & $\mathrm{V}$ & Rabat & $7.5 \times 10^{4}$ & $1+$ & $3 \min 05 \mathrm{~s}$ \\
\hline P05-13 & $\mathrm{M}, \mathrm{S}$ & 2013 & 3 & $\mathrm{MB}$ & $\mathrm{F}$ & $\mathrm{V}$ & Rabat & $1.1 \times 10^{6}$ & $\mathrm{~N}$ & - \\
\hline P06-13 & $\mathrm{F}$ & 2013 & 2 & $\mathrm{P}$ & $\mathrm{M}$ & UV & Rabat & $3.5 \times 10^{7}$ & $2+$ & $3 \min 58 \mathrm{~s}$ \\
\hline P09-13 & $\mathrm{S}$ & 2013 & 6 & MB & NA & UV & Rabat & $4.2 \times 10^{6}$ & $\mathrm{~N}$ & - \\
\hline P1-13 & I & 2013 & 4 & MB & $\mathrm{M}$ & UV & Khemisset & $1.6 \times 10^{8}$ & $1+$ & $3 \min 45 \mathrm{~s}$ \\
\hline P2-13 & $\mathrm{I}$ & 2013 & 3 & MB & $\mathrm{M}$ & UV & Khemisset & $4.6 \times 10^{9}$ & $1+$ & $3 \min 56 \mathrm{~s}$ \\
\hline P3-13 & $\mathrm{F}$ & 2013 & 3 & MB & $\mathrm{F}$ & UV & Khemisset & $5 \times 10^{4}$ & $2+$ & $1 \mathrm{~min} 36 \mathrm{~s}$ \\
\hline P4-13 & $\mathrm{I}$ & 2013 & 3 & MB & $\mathrm{F}$ & UV & Khemisset & $8.2 \times 10^{6}$ & $1+$ & $3 \min 50 \mathrm{~s}$ \\
\hline P5-13 & $\mathrm{I}$ & 2013 & 3 & MB & $\mathrm{M}$ & UV & Khemisset & $2.3 \times 10^{8}$ & $2+$ & $4 \min 25 \mathrm{~s}$ \\
\hline P6-13 & I & 2013 & 3 & MB & $\mathrm{M}$ & UV & Khemisset & $1.4 \times 10^{9}$ & $\mathrm{~N}$ & - \\
\hline P7-13 & $\mathrm{I}$ & 2013 & 3 & MB & $\mathrm{M}$ & UV & Khemisset & $1.7 \times 10^{6}$ & $2+$ & $3 \min 56 \mathrm{~s}$ \\
\hline P8-13 & $\mathrm{L}, \mathrm{S}, \mathrm{M}$ & 2013 & 3 & MB & $\mathrm{F}$ & UV & Khemisset & $6.1 \times 10^{6}$ & $1+$ & $2 \min 40 \mathrm{~s}$ \\
\hline P9-13 & $\mathrm{L}$ & 2013 & 3 & MB & $\mathrm{F}$ & UV & Khemisset & $3.4 \times 10^{6}$ & $1+$ & $7 \min 17 \mathrm{~s}$ \\
\hline P10-13 & $\mathrm{I}$ & 2013 & 3 & $\mathrm{MB}$ & $\mathrm{F}$ & UV & Khemisset & $3.3 \times 10^{9}$ & $\mathrm{~N}$ & - \\
\hline P11-13 & $\mathrm{F}$ & 2013 & 3 & MB & $\mathrm{M}$ & UV & Khemisset & $8.6 \times 10^{6}$ & $\mathrm{~N}$ & - \\
\hline P12-13 & $\mathrm{F}$ & 2013 & 6 & $\mathrm{MB}$ & $\mathrm{F}$ & UV & Khemisset & $2 \times 10^{10}$ & $2+$ & $3 \min$ \\
\hline P13-13 & $\mathrm{F}$ & 2013 & 5 & $\mathrm{MB}$ & $\mathrm{F}$ & UV & Khemisset & $4.2 \times 10^{8}$ & $\mathrm{~N}$ & - \\
\hline P14-13 & $\mathrm{F}$ & 2013 & 5 & GS & $\mathrm{M}$ & $\mathrm{V}$ & Rabat & $3.4 \times 10^{8}$ & $\mathrm{~N}$ & - \\
\hline P15-13 & $\mathrm{F}$ & 2013 & 5 & GS & $\mathrm{M}$ & $\mathrm{V}$ & Rabat & $5,3 \times 10^{8}$ & $\mathrm{~N}$ & - \\
\hline P16-13 & $\mathrm{I}$ & 2013 & 5 & St. G & $\mathrm{M}$ & UV & Rabat & $2.3 \times 10^{7}$ & $\mathrm{~N}$ & - \\
\hline P5-14 & $\mathrm{I}$ & 2014 & 6 & $\mathrm{R}$ & $\mathrm{M}$ & $\mathrm{V}$ & Rabat & $2.4 \times 10^{6}$ & $2+$ & $5 \min 41 \mathrm{~s}$ \\
\hline P6-14 & $\mathrm{F}$ & 2014 & 4 & $\mathrm{R}$ & $\mathrm{F}$ & UV & Casablanca & $1.4 \times 10^{8}$ & $2+$ & $6 \min 14 s$ \\
\hline P7-14 & $\mathrm{F}$ & 2014 & 4 & $\mathrm{H}$ & $\mathrm{M}$ & $\mathrm{V}$ & Casablanca & $4.7 \times 10^{5}$ & $1+$ & $6 \min 24 s$ \\
\hline P8-14 & $\mathrm{F}$ & 2014 & 4 & St. G & $\mathrm{F}$ & $\mathrm{V}$ & Casablanca & $2.7 \times 10^{6}$ & $\mathrm{~N}$ & - \\
\hline P9-14 & $\mathrm{I}$ & 2014 & 3 & $\mathrm{C}$ & $\mathrm{F}$ & $\mathrm{V}$ & Casablanca & $3.3 \times 10^{8}$ & $1+$ & $7 \mathrm{~min} 30 \mathrm{~s}$ \\
\hline P10-14 & $\mathrm{F}$ & 2014 & 2 & $\mathrm{BF}$ & $\mathrm{M}$ & $\mathrm{V}$ & Casablanca & $3,4 \times 10^{9}$ & $1+$ & $5 \min 05 \mathrm{~s}$ \\
\hline P11-14 & $\mathrm{F}$ & 2014 & 3 & $\mathrm{H}$ & $\mathrm{F}$ & $\mathrm{V}$ & Settat & $8 \times 10^{7}$ & $1+$ & $3 \min 56 \mathrm{~s}$ \\
\hline P12-14 & I & 2014 & 4 & $\mathrm{C}$ & $\mathrm{M}$ & $\mathrm{V}$ & Casablanca & $1.1 \times 10^{5}$ & $1+$ & $6 \min 42 s$ \\
\hline P13-14 & I & 2014 & 2 & LA & $\mathrm{M}$ & $\mathrm{V}$ & Casablanca & $6.2 \times 10^{8}$ & $2+$ & $4 \min 56 \mathrm{~s}$ \\
\hline P14-14 & $\mathrm{I}$ & 2014 & 3 & GS & $\mathrm{M}$ & $\mathrm{V}$ & Casablanca & $1,2 \times 10^{9}$ & $2+$ & $3 \min 56 \mathrm{~s}$ \\
\hline P15-14 & $\mathrm{F}$ & 2014 & 3 & GS & $\mathrm{M}$ & $\mathrm{V}$ & Casablanca & $2,8 \times 10^{6}$ & $2+$ & $5 \mathrm{~min} 30 \mathrm{~s}$ \\
\hline P16-14 & $\mathrm{F}$ & 2014 & 3 & GS & $\mathrm{M}$ & $\mathrm{V}$ & Casablanca & $1.4 \times 10^{8}$ & $1+$ & $6 \min 34 s$ \\
\hline P17-14 & $\mathrm{F}$ & 2014 & 3 & GS & $\mathrm{F}$ & $\mathrm{V}$ & Casablanca & $6.7 \times 10^{6}$ & $3+$ & $3 \min 50 \mathrm{~s}$ \\
\hline P18-14 & $\mathrm{F}$ & 2014 & 1 & MB & $\mathrm{M}$ & UV & Rabat & $1.9 \times 10^{8}$ & $2+$ & $5 \min 27 \mathrm{~s}$ \\
\hline P1-15 & $\mathrm{F}$ & 2015 & 7 & MB & $\mathrm{M}$ & UV & Rabat & $2.1 \times 10^{6}$ & $1+$ & $4 \min 19 s$ \\
\hline P2-15 & $\mathrm{F}$ & 2015 & 3 & GS & $\mathrm{M}$ & $\mathrm{V}$ & Rabat & $8.4 \times 10^{8}$ & $3+$ & $4 \min 17 \mathrm{~s}$ \\
\hline P3-15 & $\mathrm{F}$ & 2015 & 5 & GS & $\mathrm{M}$ & UV & Rabat & $9.8 \times 10^{4}$ & $2+$ & $4 \min 19 \mathrm{~s}$ \\
\hline P4-15 & $\mathrm{F}$ & 2015 & 7 & GS & $\mathrm{M}$ & $\mathrm{V}$ & Rabat & $2.1 \times 10^{6}$ & $1+$ & $4 \mathrm{~min} 30 \mathrm{~s}$ \\
\hline P5-15 & $\mathrm{F}$ & 2015 & 8 & $\mathrm{R}$ & $\mathrm{M}$ & $\mathrm{V}$ & Rabat & $3.1 \times 10^{8}$ & $1+$ & $4 \mathrm{~min} 30 \mathrm{~s}$ \\
\hline P6-15 & $\mathrm{F}$ & 2015 & 6 & MB & $\mathrm{M}$ & UV & $\begin{array}{l}\text { Machraa } \\
\text { Belaksiri }\end{array}$ & $8.8 \times 10^{4}$ & $1+$ & $4 \min 35 s$ \\
\hline
\end{tabular}




\begin{tabular}{|c|c|c|c|c|c|c|c|c|c|c|}
\hline Code & Sample & Year & $\begin{array}{l}\text { Age by } \\
\text { month }\end{array}$ & Breed & Sex & Vaccination & Origin & $\begin{array}{l}\text { Real-time } \\
\text { PCR } \\
\text { (DNA } \\
\text { copy/gm) }\end{array}$ & $\begin{array}{l}\text { COA } \\
\text { intensi } \\
\text { ty }\end{array}$ & $\begin{array}{l}\text { Reaction } \\
\text { time }\end{array}$ \\
\hline P7-15 & $\mathrm{F}$ & 2015 & 7 & MB & $\mathrm{M}$ & UV & $\begin{array}{l}\text { Machraa } \\
\text { Belaksiri } \\
\end{array}$ & $9.7 \times 10^{3}$ & $1+$ & $4 \min 40 \mathrm{~s}$ \\
\hline P8-15 & $\mathrm{F}$ & 2015 & 7 & $\mathrm{~L}$ & $\mathrm{M}$ & UV & Rabat & $2.8 \times 10^{5}$ & $1+$ & $4 \min 48 \mathrm{~s}$ \\
\hline P9-15 & $\mathrm{F}$ & 2015 & 6 & MB & $\mathrm{M}$ & UV & Rabat & $1 \times 10^{8}$ & $1+$ & $5 \mathrm{~min}$ \\
\hline P10-15 & $\mathrm{F}$ & 2015 & 3 & MB & $\mathrm{M}$ & UV & Rabat & $7.1 \times 10^{7}$ & $\mathrm{~N}$ & - \\
\hline P11-15 & $\mathrm{F}$ & 2015 & 5 & GS & $\mathrm{M}$ & UV & Rabat & $1,2 \times 10^{8}$ & $2+$ & $7 \min 12 \mathrm{~s}$ \\
\hline P12-15 & $\mathrm{F}$ & 2015 & NA & NA & NA & NA & NA & $7.8 \times 10^{6}$ & $2+$ & $10 \mathrm{~min}$ \\
\hline P13-15 & $\mathrm{F}$ & 2015 & 2 & GS & $\mathrm{M}$ & $\mathrm{V}$ & Rabat & $1.5 \times 10^{4}$ & $1+$ & $8 \min 48 s$ \\
\hline P14-15 & $\mathrm{F}$ & 2015 & 3 & $\mathrm{P}$ & $\mathrm{F}$ & UV & Rabat & $5.4 \times 10^{7}$ & $1+$ & $9 \min 10 \mathrm{~s}$ \\
\hline P15-15 & $\mathrm{F}$ & 2015 & 24 & ASD & $\mathrm{F}$ & $\mathrm{V}$ & Rabat & $6 \times 10^{7}$ & $1+$ & $7 \min 17 \mathrm{~s}$ \\
\hline P16-15 & $\mathrm{F}$ & 2015 & 8 & BS & $\mathrm{F}$ & $\mathrm{V}$ & Rabat & $5.6 \times 10^{7}$ & $2+$ & $6 \mathrm{~min}$ \\
\hline P17-15 & $\mathrm{F}$ & 2015 & 4 & GS & $\mathrm{F}$ & $\mathrm{V}$ & Rabat & $7.3 \times 10^{7}$ & $2+$ & $4 \min 28 \mathrm{~s}$ \\
\hline P18-15 & $\mathrm{F}$ & 2015 & 3 & GS & $\mathrm{M}$ & UV & Rabat & $1.2 \times 10^{5}$ & $3+$ & $1 \mathrm{~min}$ \\
\hline PC & $\mathrm{F}$ & 2015 & 5 & $\mathrm{MB}$ & $\mathrm{F}$ & UV & Rabat & $\mathrm{N}$ & $\mathrm{N}$ & - \\
\hline $\mathrm{P} 20-15$ & $\mathrm{~F}$ & 2015 & 5 & $\mathrm{P}$ & $\mathrm{F}$ & $\mathrm{V}$ & Rabat & $8.5 \times 10^{8}$ & $2+$ & $5 \min 46 s$ \\
\hline $\mathrm{P} 21-15$ & $\mathrm{~F}$ & 2015 & 1 & $\mathrm{R}$ & $\mathrm{M}$ & $\mathrm{V}$ & Rabat & $2.5 \times 10^{6}$ & $3+$ & $4 \min 16 \mathrm{~s}$ \\
\hline $\mathrm{P} 22-15$ & $\mathrm{~F}$ & 2015 & 4 & GS & $\mathrm{M}$ & UV & Rabat & $1.5 \times 10^{8}$ & $2+$ & $3 \mathrm{~min}$ \\
\hline $\mathrm{P} 23-15$ & $\mathrm{~F}$ & 2015 & NA & NA & NA & NA & Temara & $2.3 \times 10^{9}$ & $1+$ & $3 \min 20 \mathrm{~s}$ \\
\hline $\mathrm{P} 24-15$ & $\mathrm{~F}$ & 2015 & 4 & $\mathrm{R}$ & $\mathrm{M}$ & $\mathrm{V}$ & Rabat & $9.4 \times 10^{6}$ & $\mathrm{~N}$ & - \\
\hline $\mathrm{P} 25-15$ & $\mathrm{~F}$ & 2015 & 5 & St. G & $\mathrm{M}$ & UV & Rabat & $1.610^{9}$ & $1+$ & $10 \mathrm{~min}$ \\
\hline P26-15 & $\mathrm{F}$ & 2015 & 5 & St. G & $\mathrm{M}$ & UV & Rabat & $9.8 \times 10^{5}$ & $1+$ & $6 \mathrm{~min} 27 \mathrm{~s}$ \\
\hline $\mathrm{P} 27-15$ & $\mathrm{~F}$ & 2015 & 5 & St. G & $\mathrm{M}$ & UV & Rabat & $5.9 \times 10^{4}$ & $3+$ & $45 \mathrm{~s}$ \\
\hline $\mathrm{P} 28-15$ & $\mathrm{~F}$ & 2015 & 5 & St. G & $\mathrm{M}$ & UV & Rabat & $4.1 \times 10^{5}$ & $1+$ & $5 \min 26 s$ \\
\hline P29-15 & $\mathrm{F}$ & 2015 & 5 & MB & $\mathrm{M}$ & UV & Rabat & $\mathrm{N}$ & $2+$ & $3 \min 51 \mathrm{~s}$ \\
\hline P30-15 & $\mathrm{F}$ & 2015 & 2 & LR & $\mathrm{F}$ & $\mathrm{V}$ & Temara & $4.3 \times 10^{7}$ & $2+$ & $4 \min 20 \mathrm{~s}$ \\
\hline P31-15 & $\mathrm{F}$ & 2015 & 2 & LR & $\mathrm{M}$ & $\mathrm{V}$ & Temara & $5.4 \times 10^{4}$ & $1+$ & $7 \mathrm{~min} 50 \mathrm{~s}$ \\
\hline P32-15 & $\mathrm{F}$ & 2015 & 3 & LR & $\mathrm{F}$ & $\mathrm{V}$ & Temara & $2 \times 10^{6}$ & $1+$ & $5 \min 50 \mathrm{~s}$ \\
\hline P33-15 & $\mathrm{F}$ & 2015 & 2 & LR & $\mathrm{M}$ & $\mathrm{V}$ & Temara & $3.3 \times 10^{7}$ & $1+$ & $6 \min 51 \mathrm{~s}$ \\
\hline P34-15 & $\mathrm{F}$ & 2015 & 3 & MB & $\mathrm{M}$ & UV & Rabat & $2.6 \times 10^{8}$ & $2+$ & $6 \mathrm{~min}$ \\
\hline P35-15 & $\mathrm{F}$ & 2015 & 3 & MB & $\mathrm{M}$ & UV & Rabat & $1.3 \times 10^{9}$ & $2+$ & $6 \min 43 \mathrm{~s}$ \\
\hline P36-15 & $\mathrm{F}$ & 2015 & 4 & MB & $\mathrm{F}$ & UV & Rabat & $1.2 \times 10^{6}$ & $\mathrm{~N}$ & - \\
\hline P37-15 & $\mathrm{F}$ & 2015 & 4 & LR & $\mathrm{M}$ & UV & Rabat & $4.2 \times 10^{7}$ & $2+$ & $7 \mathrm{~min}$ \\
\hline P38-15 & $\mathrm{F}$ & 2015 & 4 & MB & $\mathrm{M}$ & UV & Rabat & $1.1 \times 10^{8}$ & $1+$ & $7 \min 34 s$ \\
\hline P39-15 & $\mathrm{F}$ & 2015 & 2 & LR & $\mathrm{F}$ & $\mathrm{V}$ & Temara & $6.7 \times 10^{6}$ & $1+$ & $8 \mathrm{~min}$ \\
\hline P40-15 & $\mathrm{F}$ & 2015 & 2 & St. G & $\mathrm{M}$ & $\mathrm{V}$ & Temara & $2.1 \times 10^{6}$ & $2+$ & $4 \min 55 \mathrm{~s}$ \\
\hline P41-15 & $\mathrm{F}$ & 2015 & 2 & St. G & $\mathrm{M}$ & $\mathrm{V}$ & Temara & $5.4 \times 10^{5}$ & $\mathrm{~N}$ & - \\
\hline $\mathrm{P} 42-15$ & $\mathrm{~F}$ & 2015 & 3 & MB & $\mathrm{M}$ & UV & Rabat & $4.9 \times 10^{8}$ & $\mathrm{~N}$ & - \\
\hline P43-15 & $\mathrm{F}$ & 2015 & 3 & $\mathrm{MB}$ & $\mathrm{M}$ & UV & Rabat & $6.2 \times 10^{7}$ & $1+$ & $10 \mathrm{~min}$ \\
\hline P44-15 & $\mathrm{F}$ & 2015 & 2 & $\mathrm{BS}$ & $\mathrm{F}$ & UV & Temara & $1.8 \times 10^{5}$ & $1+$ & $10 \mathrm{~min}$ \\
\hline $\mathrm{P} 45-15$ & $\mathrm{~F}$ & 2015 & 2 & $\mathrm{BS}$ & $\mathrm{F}$ & UV & Temara & $5.2 \times 10^{5}$ & $2+$ & $3 \min 57 \mathrm{~s}$ \\
\hline P46-15 & $\mathrm{F}$ & 2015 & 2 & $\mathrm{BS}$ & $\mathrm{F}$ & UV & Temara & $9.9 \times 10^{5}$ & $2+$ & $4 \min 52 \mathrm{~s}$ \\
\hline P47-15 & $\mathrm{F}$ & 2015 & 7 & MB & $\mathrm{M}$ & UV & Rabat & $1.7 \times 10^{6}$ & $\mathrm{~N}$ & - \\
\hline P48-15 & $\mathrm{F}$ & 2015 & 4 & BS & $\mathrm{M}$ & UV & Rabat & $1.1 \times 10^{7}$ & $3+$ & $3 \min 58 \mathrm{~s}$ \\
\hline PA & $\mathrm{F}$ & 2015 & 1 & $\mathrm{R}$ & $\mathrm{F}$ & $\mathrm{V}$ & Sale & $\mathrm{N}$ & $2+$ & $4 \min 27 \mathrm{~s}$ \\
\hline
\end{tabular}

M: Male ; F: Female ; I: Intestine ; L: Liver ; S: Spleen ; M: Myocardium ; F: Feces ; V: Vaccinated ; UV: Unvaccinated ; NA: No information available ; ASD: Atlas Shepherd Dog ; BS: Belgian Shepherd ; BF: bichon frise ; C: Chihuahua ; GR: Golden retriever ; GS: German shepherd ; H: Husky ; LA: Lhassa Apso ; LR: Labrador Retriever ; MB: Mixed breed ; P: Poodle ; R: Rottweiler ; St. G: Saint-Germain Pointing Dog ;N: Negative; 1+ : low intensity; 2+: median intensity; $3+$ : high intensity 


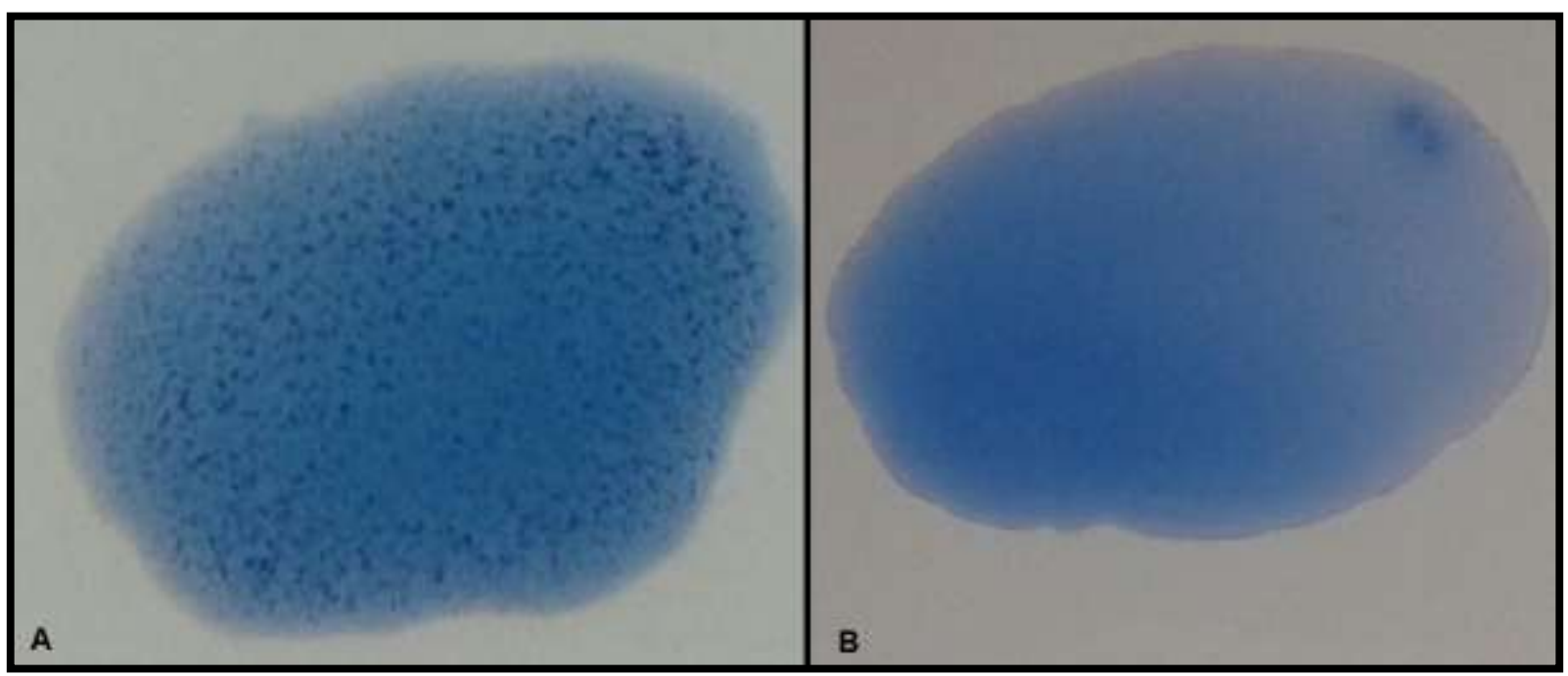

Figure 2:-Coagglutination test after mixing the reagent with positive and negative samples; A: Agglutinin particles in positive reaction; B: negative reaction

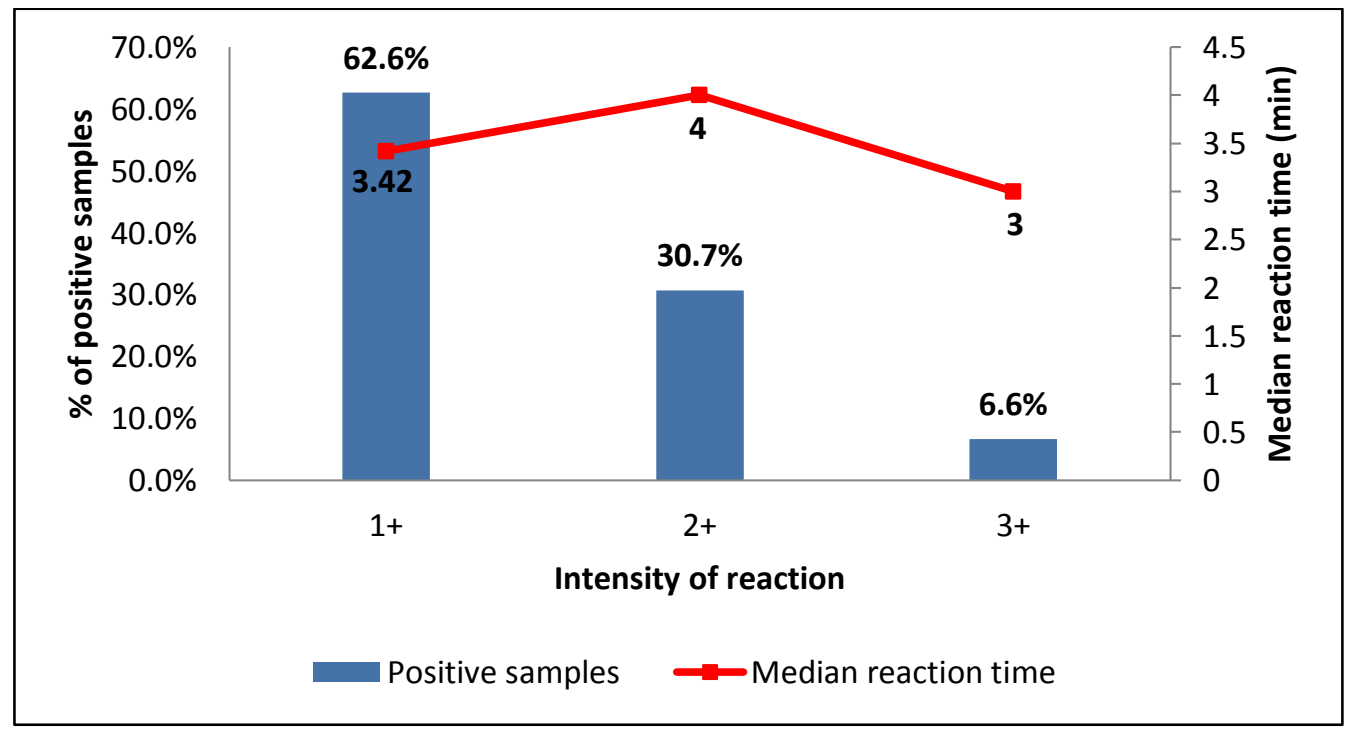

Figure 3:-Intensity of reaction in positive samples and average of the scored reaction time (1+: low reaction, 2+: medium reaction, 3+: High reaction)

\section{Discussion:-}

Rapid diagnosis of canine parvovirus remains the first important step to adopt the appropriate measures for disease control and transmission reduction especially in kennels. Molecular based diagnostic methods were demonstrated specific and sensitive (Decaro et al., 2005b; Desario et al., 2005) but cannot be performed in routine veterinary practice as these assays require skill and expensive equipment. The heamagglutination test was described as a reference test in labs that are not equipped with molecular platforms (Amrani et al., 2016; Desario et al., 2005) (Amrani et al., 2016). Nevertheless the difficulty in obtaining fresh pig erythrocytes and the poor correlation with PCR (Desario et al., 2005) hampers the use of this test in routine veterinary practice. Thus, the development of an inexpensive, simple, rapid, specific, sensitive and easy to use diagnostic test in veterinary practice is useful. The coagglutination test was previously performed for CPV-2 (Genovn and Ivanov, 2006) and diverse pathogens detection (Joshi et al., 1989; Montassier et al., 1994; Yoshimizu and Kimura, 1985). We investigated the potential use of COA to detect CPV-2 in clinical samples and evaluated its performances in comparison with the PCR test.

Previous COA assays required dark background or using microscope (x10) to be read macroscopically (Yoshimizu and Kimura, 1985) with the possibility to affect the test lecture and to determine its intensity. Thus, COA using 
methylen blue stained reagent provides an easy alternative and more objective lecture without the use of a microscope. A good agreement $(80.2 \%)$ was found between COA and PCR as a gold standard test. In addition, no false positive samples was observed with COA test compared with haemagglutination test which was shown to be poorly sensitive, resulting in a high proportion of false-negative results. Hence, the COA test developed in the present study can be considered as a good alternative for use as a bench or penside test in clinical veterinary practice.

\section{Summary:-}

We report in the current study the development of a, simple, reliable and rapid test to detect canine parvovirus in daily veterinary practice. High performances, processing of many samples, easy procedure and results interpretation make the COA an effective tool especially in field conditions and laboratories that are not equipped with molecular technology platform.

\section{References:-}

1. Amrani, N., Desario, C., Kadiri, A., Cavalli, A., Berrada, J., Zro, K., Sebbar, G., Colaianni, M.L.M.L., Parisi, A., Elia, G., Buonavoglia, C., Malik, J., Decaro, N., 2016. Molecular epidemiology of canine parvovirus in Morocco. Infect. Genet. Evol. 41, 201-206. https://doi.org/10.1016/j.meegid.2016.04.005

2. Decaro, N., Buonavoglia, C., 2012. Canine parvovirus-A review of epidemiological and diagnostic aspects, with emphasis on type 2c. Vet. Microbiol. 155, 1-12. https://doi.org/10.1016/j.vetmic.2011.09.007

3. Decaro, N., Desario, C., Elia, G., Martella, V., Mari, V., Lavazza, A., Nardi, M., Buonavoglia, C., 2008. Evidence for immunisation failure in vaccinated adult dogs infected with canine parvovirus type 2c. New Microbiol. 31, $125-130$.

4. Decaro, N., Desario, C., Parisi, A., Martella, V., Lorusso, A., Miccolupo, A., Mari, V., Colaianni, M.L., Cavalli, A., Di Trani, L., Buonavoglia, C., 2009. Genetic analysis of canine parvovirus type 2c. Virology 385, 5-10. https://doi.org/10.1016/j.virol.2008.12.016

5. Decaro, Campolo, M., Desario, C., Elia, G., Martella, V., Lorusso, E., Buonavoglia, C., 2005a. Maternally-derived antibodies in pups and protection from canine parvovirus infection. Biologicals 33, 261-267. https://doi.org/10.1016/j.biologicals.2005.06.004

6. Decaro, Elia, G., Martella, V., Desario, C., Campolo, M., Trani, L. Di, Tarsitano, E., Tempesta, M., Buonavoglia, C., 2005b. A real-time PCR assay for rapid detection and quantitation of canine parvovirus type 2 in the feces of dogs. Vet. Microbiol. 105, 19-28. https://doi.org/10.1016/j.vetmic.2004.09.018

7. Desario, C., Decaro, N., Campolo, M., Cavalli, A., Cirone, F., Elia, G., Martella, V., Lorusso, E., Camero, M., Buonavoglia, C., 2005. Canine parvovirus infection: Which diagnostic test for virus? J. Virol. Methods 126, $179-185$. https://doi.org/10.1016/j.jviromet.2005.02.006

8. Genovn, K., Ivanov, I., 2006. Bulgarian Academy of Sciences A rapid test for detection of canine parvovirus 45-48.

9. Joshi, R.K., Chandra, R., Rao, V.D.P., Garg, S.K., 1989. COAGGLUTINATION TEST : A SIMPLE AND RAPID DIAGNOSTIC TECHNIQUE FOR GOAT POX 233-235.

10. Kantere, M.C., Athanasiou, L. V, Spyrou, V., Kyriakis, C.S., Kontos, V., Chatzopoulos, D.C., Tsokana, C.N., Billinis, C., 2015. Diagnostic performance of a rapid in-clinic test for the detection of Canine Parvovirus under different storage conditions and vaccination status. J Virol Methods 215-216, 52-55. https://doi.org/10.1016/j.jviromet.2015.02.012

11. Kim, Y.K., Lim, S.-I., Choi, S., Cho, I.-S., Park, E.-H., An, D.-J., 2015. A novel assay for detecting canine parvovirus using a quartz crystal microbalance biosensor. J. Virol. Methods 219, $23-7$. https://doi.org/10.1016/j.jviromet.2015.03.015

12. Montassier, H.J.J., Araújo Júnior, J.P., Pinto, A.. A., Araujo, J.P., Pinto, A.. A., 1994. Rapid coagglutination test for the detection and typing of foot and mouth disease virus. J. Virol. Methods 50, 29-42.

13. Prittie, J., 2004. Canine parvoviral enteritis: a review of diagnosis, management, and prevention. J. Vet. Emerg. Crit. Care 14, 167-176. https://doi.org/10.1111/j.1534-6935.2004.04020.x

14. Rakha, G.M.H., Abdl-Haleem, M.M., Farghali, H.A.M., Abdel-Saeed, H., 2015. Prevalence of common canine digestive problems compared with other health problems in teaching veterinary hospital, Faculty of Veterinary Medicine, Cairo University, Egypt. Vet. world 8, 403-11. https://doi.org/10.14202/vetworld.2015.403-411

15. Singh, B.R., Yadav, R.C., Singh, S.P., Sharma, V.D., 1998. Coagglutination test: A simple and rapid immunodiagnostic test for Parvovirus infection in dogs. Indian J. Exp. Biol. 36, 622-624.

16. Ylmaz, Z., Kennerman, E., Senturk, S., Temizel, M., Aytug, N., 2002. The evaluation of cats and dogs referred to the clinics of companion animal-Internal Medicine, Veterinary Faculty, University of Uludag (1990-2000). Vet. Fak. Dergisi, Uludag Univ. 21, 23-31.

17. Yoshimizu, M., Kimura, T., 1985. A coagglutination test with antibody-sensitized staphylococci for rapid and simple diagnosis of bacterial and viral diseases of fish. Fish Pathol. 20, 243-261. https://doi.org/10.3147/jsfp.20.243 